University of Nebraska - Lincoln

DigitalCommons@University of Nebraska - Lincoln

Faculty Publications from the Department of Engineering Mechanics

2009

\title{
Reduction of surface acoustic wave resonator normal acceleration sensitivity using piezoelectric actuators
}

\author{
X. Y. Shan \\ Huazhong University of Science and Technology, Wuhan \\ Y. T. Hu \\ Huazhong University of Science and Technology, Wuhan \\ J. S. Yang \\ University of Nebraska-Lincoln, jyang1@unl.edu
}

Follow this and additional works at: https://digitalcommons.unl.edu/engineeringmechanicsfacpub

Part of the Mechanical Engineering Commons

Shan, X. Y.; Hu, Y. T.; and Yang, J. S., "Reduction of surface acoustic wave resonator normal acceleration sensitivity using piezoelectric actuators" (2009). Faculty Publications from the Department of Engineering Mechanics. 64.

https://digitalcommons.unl.edu/engineeringmechanicsfacpub/64

This Article is brought to you for free and open access by the Mechanical \& Materials Engineering, Department of at DigitalCommons@University of Nebraska - Lincoln. It has been accepted for inclusion in Faculty Publications from the Department of Engineering Mechanics by an authorized administrator of DigitalCommons@University of Nebraska - Lincoln. 


\title{
Reduction of surface acoustic wave resonator normal acceleration sensitivity using piezoelectric actuators
}

\author{
X. Y. Shan, ${ }^{1}$ Y. T. Hu, ${ }^{1}$ and J. S. Yang ${ }^{2, a)}$ \\ ${ }^{1}$ School of Civil Engineering and Mechanics, Huazhong University of Science and Technology, Wuhan, \\ Hubei 430074, People's Republic of China \\ ${ }^{2}$ Department of Engineering Mechanics, University of Nebraska, Lincoln, Nebraska 68588-0526, USA
}

(Received 20 August 2009; accepted 3 September 2009; published online 23 September 2009)

\begin{abstract}
We attach a piezoelectric actuator to a surface acoustic wave resonator under normal acceleration to reduce its acceleration sensitivity. It is shown theoretically that under a given acceleration, the acceleration induced frequency shift in the resonator can be reduced or even completely eliminated when a proper electric field is applied to the piezoelectric actuator. () 2009 American Institute of Physics. [doi:10.1063/1.3236750]
\end{abstract}

Crystal resonators are key components of oscillators for frequency generation and operation with extensive applications in time keeping, telecommunication, sensing, and control. These devices are often mounted on objects in motion, e.g., helicopters, missiles, and satellites. Resonators are usually made of crystal plates. They operate with either bulk acoustic waves or surface acoustic waves (SAW). When a resonator is under acceleration, the inertial force due to the acceleration causes initial or biasing deformations and stresses in the resonator, which produce frequency shifts in the resonator. For the above applications, resonator acceleration sensitivity needs to be minimized or, ideally, eliminated through design. The study of resonator acceleration sensitivity has been a key topic and is of current interest for both military and civilian applications. ${ }^{1-4}$ At present existing technologies allow the reduction of resonator acceleration sensitivity to about $10^{-10} / \mathrm{g}$. The goal of the U.S. Army Communication and Electronics Command has been to further reduce it to $10^{-12} / \mathrm{g}$ in the not-too-distant future. However, the progress has been slow and there are recent results indicating the possible existence of a lower bound of resonator acceleration sensitivity with present technologies. ${ }^{5,6} \mathrm{New}$ approaches are needed for further, significant reduction to meet the military need.

Recently it has been proposed to apply a biasing electric field to a quartz resonator to use the electric field induced deformation to reduce the acceleration-frequency effect. ${ }^{7}$ Quartz is a material with very weak electromechanical coupling. Hence, the electric field induced deformation is very small. More recently, it has also been suggested ${ }^{8,9}$ that a layer of a piezoelectric material with strong electromechanical coupling, e.g., polarized ceramics, be attached to a crystal resonator to enhance the electric field induced deformation for electric field sensing. Motivated by Refs. 7-9, we propose to attach a piezoelectric layer to a crystal plate SAW resonator for the reduction of its normal acceleration sensitivity. The piezoelectric layer serves as an actuator. Under a given acceleration, a proper electric field is determined and applied to the piezoelectric actuator to induce deformation in the structure to reduce the effect of the acceleration induced deformation and thereby reducing the resonator acceleration sensitivity. Specifically, we perform a theoretical analysis on
SAW silicon and germanium resonators on a piezoelectric ceramic layer under a normal acceleration to demonstrate the idea.

Consider a homogeneous material body occupying a region $V$. The body is free from any deformations and fields. The mass density is $\rho_{0}$. The second- and third-order elastic constants are $c_{K \alpha L \gamma}$ and $c_{K \alpha L \gamma A B}$, respectively. Suppose that the governing equations and boundary conditions allow a linear acoustic mode with frequency $\omega$ and displacement $u_{\alpha}$. When an initial displacement field $\mathbf{w}$ with initial stress $\mathbf{T}^{0}$ and initial strain $S_{K L}^{0}=\left(w_{K, L}+w_{L, K}\right) / 2$ is applied, the frequency of the mode is perturbed a little and is denoted by $\omega+\Delta \omega$. The frequency shift due to the initial fields is represented by the following integral from a first-order perturbation analysis, ${ }^{10}$

$$
\frac{\Delta \omega}{\omega} \cong \frac{1}{2 \omega^{2}} \frac{\int_{V} \hat{c}_{L \gamma M \alpha} u_{\gamma, L} u_{\alpha, M} d V}{\int_{V} \rho_{0} u_{\alpha} u_{\alpha} d V},
$$

where $\hat{c}_{K \alpha L \gamma}$ is the modification of the second-order elastic constants by the initial fields and is given by ${ }^{10}$

$$
\hat{c}_{K \alpha L \gamma}=T_{K L}^{0} \delta_{\alpha \gamma}+c_{K \alpha L N} w_{\gamma, N}+c_{K M L \gamma} w_{\alpha, M}+c_{K \alpha L \gamma A B} S_{A B}^{0} .
$$

In order to use Eq. (1), two things are needed. One is the initial field $\mathbf{w}$ which in our case is induced by acceleration. The other is the unperturbed mode $u_{\alpha}$ in the absence of the initial fields. They will be determined separately in the following.

We determine the acceleration induced biasing deformation first. Consider the two-layered plate under normal acceleration as shown in Fig. 1. The plate is assumed to be thin in the sense that its in-plane dimension is much larger than its

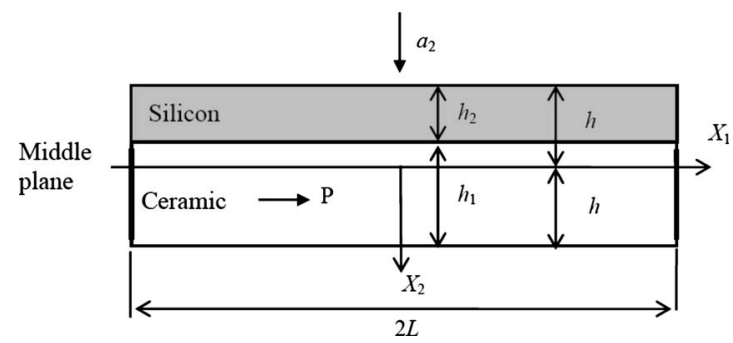

FIG. 1. A crystal plate with a piezoelectric layer. 
total thickness $(2 L \gg 2 h)$. The thickness of the silicon layer is much larger than the wavelength of the SAWs we are considering so that the SAWs at the top of the silicon layer do not feel the interface between the two layers. The ceramic layer is poled in the $X_{1}$ direction. It is electroded at both ends and an axial electric field is applied. We consider plane-strain (cylindrical) deformation of the plate. Since the combined plate is asymmetric about its middle plane, the middle plane experiences both extension and flexure. We use the coupled theory for extension and elementary (Kirchhoff) flexure. ${ }^{11}$ Let $w_{2}^{(0)}$ and $w_{1}^{(0)}$ be the middle plane flexural and extensional displacements and $N, M$, and $Q$ be the plate extensional force, bending moment, and transverse shear force, respectively. In the case of static and plane-strain deformation, the relevant equations of motion take the following form: ${ }^{11}$

$$
\begin{aligned}
& N_{, 1}=0, \\
& Q_{, 1}=\left(\rho_{1} h_{1}+\rho_{2} h_{2}\right) a_{2}, \\
& Q=M_{, 1} .
\end{aligned}
$$

Equation (3a) is for extension. Eq. (3b) is for flexure. Eq. (3c) is a moment equation which provides the shear forcebending moment relation in the Kirchhoff theory. The plate constitutive equations connecting the plate resultants and the plate displacements are given by

$$
\begin{aligned}
& N=\int_{-h}^{h} T_{11} d X_{2}=a_{11} w_{1,1}^{(0)}-h_{1} e_{11}^{p} E_{1}+b_{11} w_{2,11}^{(0)}, \\
& M=\int_{-h}^{h} T_{11} X_{2} d x_{2}=-\gamma_{11} w_{2,11}^{(0)}-b_{11} w_{1,1}^{(0)}-\frac{h_{1} h_{2}}{2} e_{11}^{p} E_{1},
\end{aligned}
$$

where

$$
\begin{aligned}
& a_{11}=h_{1} c_{11}^{p}+h_{2} \bar{c}_{11}, \quad b_{11}=-\frac{1}{2}\left(c_{11}^{p}-\bar{c}_{11}\right) h_{1} h_{2}, \\
& \gamma_{11}=\frac{1}{12} c_{11}^{p}\left(h_{1}^{3}+3 h_{1} h_{2}^{2}\right)+\frac{1}{12} \bar{c}_{11}\left(h_{2}^{3}+3 h_{2} h_{1}^{2}\right), \\
& \bar{c}_{11}=c_{11}-c_{12}^{2} / c_{22}, \\
& c_{11}^{p}=c_{33}^{E}-\left(c_{13}^{E}\right)^{2} / c_{11}^{E}, \quad e_{11}^{p}=e_{33}-e_{31} c_{13}^{E} / c_{11}^{E} .
\end{aligned}
$$

For flexure we consider a simply-supported plate without end deflection and end moments. For extension we consider the case when the two ends are free without axial forces. To make the extensional displacement field unique, we consider the case when the plate center has no axial displacement. Then the plate extensional and flexural displacements under the acceleration $a_{2}$ and the applied electric field $E_{1}$ are found to be

$$
\begin{aligned}
w_{1}^{(0)}= & A e_{11}^{P} E_{1} X_{1}-b_{11} C L^{2} X_{1}+\frac{1}{3} b_{11} C X_{1}^{3} \\
w_{2}^{(0)}= & \frac{1}{2} B e_{11}^{P} E_{1} X_{1}^{2}+\frac{1}{2} a_{11} C L^{2} X_{1}^{2}-\frac{1}{12} a_{11} C X_{1}^{4}-\frac{1}{2} B e_{11}^{P} E_{1} L^{2} \\
& -\frac{5}{12} a_{11} C L^{4}
\end{aligned}
$$

where

$$
\begin{gathered}
A=\frac{h_{1} \gamma_{11}+\frac{1}{2} h_{1} h_{2} b_{11}}{a_{11} \gamma_{11}-b_{11}^{2}}, \quad B=\frac{-\frac{1}{2} h_{1} h_{2} a_{11}-h_{1} b_{11}}{a_{11} \gamma_{11}-b_{11}^{2}}, \\
C=\frac{\frac{1}{2}\left(\rho_{1} h_{1}+\rho_{2} h_{2}\right) a_{2}}{a_{11} \gamma_{11}-b_{11}^{2}} .
\end{gathered}
$$

Then the displacement field $\mathbf{w}$ can be calculated from the plate extension and flexure through

$$
\begin{aligned}
& w_{1} \cong w_{1}^{(0)}\left(X_{1}\right)-X_{2} w_{2,1}^{(0)}, \\
& w_{2} \cong w_{2}^{(0)}, \\
& w_{3}=0 .
\end{aligned}
$$

The displacement gradients needed in the perturbation integral can be calculated as follows. $w_{1,1}$ and $w_{1,2}$ can be directly obtained from differentiating Eq. (9a). $w_{2,1}$ can be obtained from differentiating Eq. (9b). $w_{2,2}$ is obtained from the stress relaxation condition $T_{2}=0$ for thin plates.

Next we determine the unperturbed SAW modes. The SAWs are propagating along the top surface of the silicon plate in Fig. 1. Since the wavelength of the SAWs is much shorter than the thickness of the silicon plate, to the SAWs the silicon plate is effectively a half space. We consider Rayleigh surface waves with frequency $\omega$, wave number $\xi$ in the $\bar{X}_{1}$ direction, phase speed $V_{R}=\omega / \xi$, and displacement $u_{\alpha}$. We are interested in silicon and germanium which are of cubic symmetry $(\mathrm{m} 3 \mathrm{~m})$ and allow plane-strain motions with $u_{3}=0$ and $\partial_{3}=0$. Rayleigh waves over a half space of $m 3 m$ crystals with a traction-free boundary surface are given by ${ }^{12}$

$$
\begin{aligned}
u_{1}= & \exp \left(-2 \pi \beta \bar{X}_{2} / \lambda_{R}\right) \cos \left(2 \pi g \frac{\bar{X}_{2}}{\lambda_{R}}+\alpha\right) \\
& \times \cos \left[\omega\left(t-\frac{\bar{X}_{1}}{V_{R}}\right)-\alpha\right], \\
u_{2}= & -r \exp \left(-2 \pi \beta \bar{X}_{2} / \lambda_{R}\right) \cos \left(2 \pi g \frac{\bar{X}_{2}}{\lambda_{R}}-\alpha\right) \\
& \times \sin \left[\omega\left(t-\frac{\bar{X}_{1}}{V_{R}}\right)-\alpha\right],
\end{aligned}
$$

where $\beta, \lambda_{R}, g, \alpha, V_{R}$, and $r$ depend on material parameters. $\bar{X}_{1}=X_{1}$ and $\bar{X}_{2}=X_{2}+h$. For use in Eq. (1), we need to expand Eq. (10) into cos $\omega t$ and sin $\omega t$ terms and use the coefficient of either $\cos \omega t$ or $\sin \omega t$.

With the initial fields in Eqs. (9) and the unperturbed modes in Eq. (10), we are ready to calculate the perturbation integral in Eq. (1). We are interested in surface waves with frequencies around $f=1 \mathrm{GHz}$. The corresponding wavelength is $\lambda=4.92 \times 10^{-3} \mathrm{~mm}$ for silicon and 2.92 $\times 10^{-3} \mathrm{~mm}$ for germanium. For geometric parameters the thickness of the silicon or germanium layer is of the order of $0.1 \mathrm{~mm}$ which is much larger than the SAW wavelength. For the piezoelectric substrate we use lead zirconate titanate- $5 \mathrm{H}$ (PZT-5H). For silicon and germanium, the wave parameters in Eq. (10) can be determined as

$$
\begin{gathered}
V_{R}=4917 \mathrm{~m} / \mathrm{s}, \quad g=0.4808, \quad \beta=0.4556, \\
r=1.226, \quad \alpha=58^{\circ} 1^{\prime},
\end{gathered}
$$




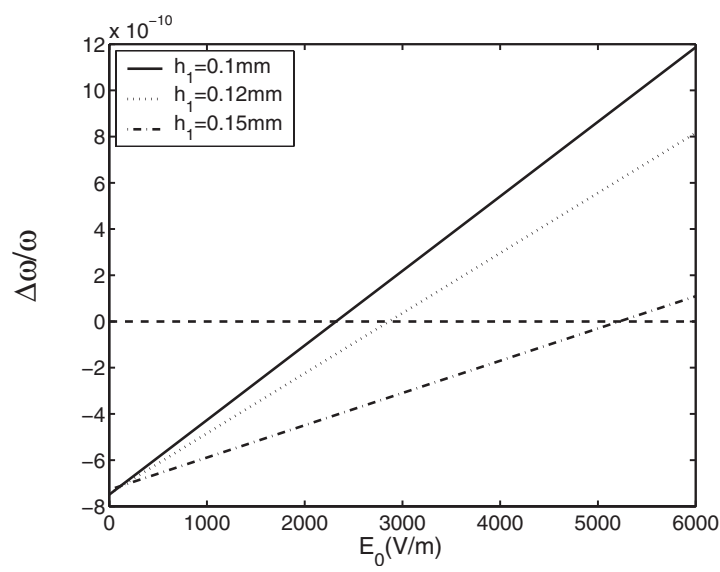

FIG. 2. Frequency shift vs electric field in silicon $\left(a_{2}=g\right.$ and $\left.h_{2}=0.1 \mathrm{~mm}\right)$.

$$
\begin{gathered}
V_{R}=2924 \mathrm{~m} / \mathrm{s}, \quad g=0.5122, \quad \beta=0.4378, \\
r=1.1938, \quad \alpha=55^{\circ} 53^{\prime} .
\end{gathered}
$$

Figures 2 and 3 show frequency shifts versus electric field $\left(E_{1}=E_{0}\right)$ under $a_{2}=g=9.81 \mathrm{~m} / \mathrm{s}^{2}$ in a silicon resonator and a germanium resonator, respectively. A linear relationship between the frequency shift and the electric field is predicted. The most important result of this paper is that under a particular electric field the frequency shift vanishes. Therefore, theoretically, the acceleration sensitivity can be completely eliminated using this technique. In real applications, an accelerometer is needed to detect the acceleration and a control unit is needed to apply a proper electric field according to the acceleration.

Figures 2 and 3 also show that the proper electric field for a zero frequency shift is sensitive to the relative thickness

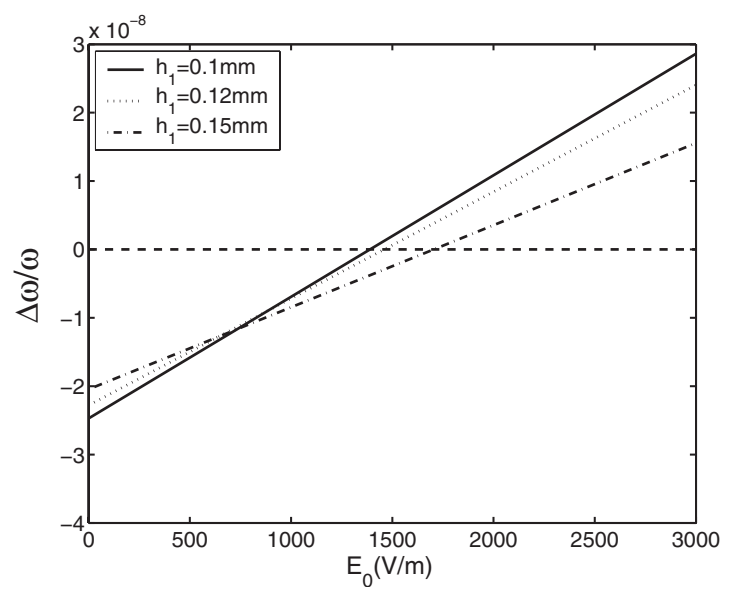

FIG. 3. Frequency shift vs electric field in germanium $\left(a_{2}=g\right.$ and $h_{2}$ $=0.1 \mathrm{~mm}$ ). of the two layers. The effect of the layer thicknesses is complicated. Under a positive $a_{2}$, the inertial force points up along $-X_{2}$. In this case the top surface of the silicon or germanium layer is stretched. When a positive electric field is applied to the ceramic layer, since for PZT-5H the relevant piezoelectric strain constant $d_{33}$ is positive, the piezoelectric layer tends to elongate which induces both bending and extension of the two-layered plate. The electric-field-induced bending reduces the acceleration-induced stretch at the top surface of the silicon layer and hence reduces the acceleration sensitivity but the extension does the opposite. Therefore there are two different situations depending on whether the piezoelectric layer is relatively thick or not. When the piezoelectric layer is not thick, its tendency to elongate mainly induces bending of the two-layered plate which is helpful for acceleration reduction. When the piezoelectric layer is thick, its elongation tends to stretch the two layered plate in addition to producing bending. This stretching effect is countering some of the bending effect and therefore the reduction of the frequency shift becomes less effective.

In summary, we have shown that the normal acceleration sensitivity of a SAW resonator with an attached piezoelectric layer under an applied electric field depends on the electric field linearly. This effect can be used to reduce the normal acceleration sensitivity of the resonator. With a proper electric field the acceleration sensitivity can be completely eliminated.

The work was supported by the National Science Foundation of China (Grant No. 10872074) and Major State Basic Research Development Program of China (973 Program) (Grant No. 2009CB724205).

${ }^{1}$ H. F. Tiersten and Y. S. Zhou, J. Appl. Phys. 70, 4708 (1991).

${ }^{2}$ H. F. Tiersten and D. V. Shick, J. Appl. Phys. 67, 60 (1990).

${ }^{3}$ J. A. Kosinski and R. A. Pastore, Jr., IEEE Trans. Ultrason. Ferroelectr. Freq. Control 48, 1426 (2001).

${ }^{4}$ J. S. Yang and S. H. Guo, Appl. Phys. Lett. 88, 193509 (2006).

${ }^{5}$ J. A. Kosinski, R. A. Pastore, Jr., and J. S. Yang, Proceedings of the IEEE Frequency Control Symposium, Kansas City, Missouri, 7-9 June 2000 (IEEE, Piscataway, 2000), p. 345.

${ }^{6}$ J. S. Yang and S. H. Guo, IEEE Trans. Ultrason. Ferroelectr. Freq. Control 53, 1562 (2006).

${ }^{7}$ Y.-K. Yong and M. S. Patel, Int. J. Appl. Electromagn. Mech. 22, 69 (2005).

${ }^{8}$ Y. Y. Zhou, W. Q. Chen, J. S. Yang, and J. K. Du, "Thickness-shear vibration of a quartz plate connected to piezoelectric plates and electric field sensing," Ultrasonics (to be published).

${ }^{9}$ Y. Y. Zhou, J. S. Yang, and W. Q. Chen, "Surface waves propagating in an elastic-piezoelectric bilayer plate and electric field sensing," IEEE Sens. J. (to be published).

${ }^{10}$ H. F. Tiersten, J. Acoust. Soc. Am. 64, 832 (1978).

${ }^{11}$ J. S. Yang, H. G. Zhou, and S. X. Dong, IEEE Trans. Ultrason. Ferroelectr. Freq. Control 53, 456 (2006).

${ }^{12}$ R. Stoneley, Proc. R. Soc. London, Ser. A 232, 447 (1955). 